\title{
ON THE EXACT SOLUTION ON A LINEAR DIFFERENTIAL EQUATION OF FIRST ORDER
}

\author{
Lazo A. Dimov ${ }^{1}$, Boro M. Piperevski ${ }^{2}$, Elena Hadzieva ${ }^{3}$
}

Abstract. We are considering the linear differential equation of first order

$$
f(x) y^{\prime}-f^{\prime}(x) y=f^{3}(x) R(x, t), t=\sqrt{a_{4} x^{4}+a_{3} x^{3}+a_{2} x^{2}+a_{1} x+a_{o}},
$$

where $R(x, t)$ is a rational function. We are giving a condition for the equation to have exact solution and a method for finding the solution.

\section{INTRODUCTION}

It is known that the linear differential equation of first order

$$
f(x) y^{\prime}-f^{\prime}(x) y=f^{3}(x) R(x, t),
$$

has a general solution that can be written in the quadrature form

$$
y=f(x)\left[\int f(x) R(x, t(x)) d x+C\right] .
$$

If $f(x)$ is a rational function, then the integral on the right in (2) is elliptic. Its primitive function is possible to be found only if the integral is pseudo elliptic.

Namely, the integrals of the form

$$
\int R\left(x, \sqrt{x^{4}+p x^{2}+q x+r}\right) d x
$$

that can be determined as elliptic functions are called pseudoeliptic integrals.

It is known that the fourth degree polynomial

$$
p_{4}(x)=a_{0} x^{4}+a_{1} x^{3}+a_{2} x^{2}+a_{3} x+a_{4}
$$

can be transformed into the polynomial

$$
p_{4}(y)=a_{0}\left(y^{4}+p y^{2}+q y+r\right)
$$

using the substitution

$$
x=y-\frac{a_{1}}{4 a_{0}} .
$$

The coefficients $p, q, r$ depend on the coefficients $a_{0}, a_{1}, a_{2}, a_{3}$ and $a_{4}$. Without loss of generality, we'll consider the polynomial

$$
p(x)=x^{4}+p x^{2}+q x+r .
$$

2010 Mathematics Subject Classification. Primary: 34A05, Secondary: 11D25

Key words and phrases. linear ordinary differential equation of first order, general solution, exact solution, pseudoeliptic integral 
According to the famous Girolamo Kardano's (1501-1576) formulae for solving algebraic equation of fourth degree, the polynomial (4) can be represented in the form

$$
\begin{aligned}
p(x) & =x^{4}+p x^{2}+q x+r= \\
& =\left[x^{2}-u x+\frac{1}{2}\left(u^{2}+p+\frac{q}{u}\right)\right] \cdot\left[x^{2}+u x+\frac{1}{2}\left(u^{2}+p-\frac{q}{u}\right)\right] \\
& =\left[\left(x-\frac{u}{2}\right)^{2}+\frac{1}{2}\left(\frac{u^{2}}{2}+p+\frac{q}{u}\right)\right] \cdot\left[x^{2}+u x+\frac{1}{2}\left(u^{2}+p-\frac{q}{u}\right)\right] .
\end{aligned}
$$

Supposing that the polynomial (4) has a double root $x=\frac{u}{2}$, the relation

$$
u^{3}+2 p u+2 q=0,
$$

is true. Now, the polynomial takes the form

$$
p(x)=x^{4}+p x^{2}+q x+r=\left(x-\frac{u}{2}\right)^{2}\left[\left(x+\frac{u}{2}\right)^{2}-\frac{q}{u}\right] .
$$

\section{THE MAIN RESULT}

At this point we will discuss on a class of pseudo elliptic integrals. Assuming that the polynomial under the square root has double root $x=\frac{u}{2}$, we will prove that the integral can be reduced to the integral of rational function. Namely, if the polynomial (1.4) has a double root $x=\frac{u}{2}$, then (1.6) is true and for the integral the following is obtained:

$$
\begin{aligned}
\int R\left(x, \sqrt{x^{4}+p x^{2}+q x+r}\right) d x & \left.=\int R\left\{x, \sqrt{\left(x-\frac{u}{2}\right)^{2}\left[\left(x+\frac{u}{2}\right)^{2}-\frac{q}{u}\right.}\right]\right\} d x \\
& =\int R\left\{x,\left(x-\frac{u}{2}\right) \sqrt{\left(x+\frac{u}{2}\right)^{2}-\frac{q}{u}}\right\} d x .
\end{aligned}
$$

We will introduce the new variable $s$ by Euler's substitution in the last integral,

$$
\sqrt{\left(x+\frac{u}{2}\right)^{2}-\frac{q}{u}}=x+\frac{u}{2}-s
$$

which transforms the original integral in the integral of rational function of variable $s$. Namely,

$$
\begin{gathered}
\left(x+\frac{u}{2}\right)^{2}-\frac{q}{u}=\left(x+\frac{u}{2}-s\right)^{2},\left(x+\frac{u}{2}\right)^{2}-\frac{q}{u}=\left(x+\frac{u}{2}\right)^{2}-2 s\left(x+\frac{u}{2}\right)+s^{2}, \\
x+\frac{u}{2}=\frac{s^{2}+\frac{q}{u}}{2 s}, d x=\frac{s^{2}-\frac{q}{u}}{2 s^{2}} d s, \sqrt{\left(x+\frac{u}{2}\right)^{2}-\frac{q}{u}}=\frac{s^{2}+\frac{q}{u}}{2 s}-s=\frac{-s^{2}+\frac{q}{u}}{2 s},
\end{gathered}
$$

which yields to:

$$
\begin{aligned}
\int R\left\{x,\left(x-\frac{u}{2}\right) \sqrt{\left(x+\frac{u}{2}\right)^{2}-\frac{q}{u}}\right\} d x & =\int R\left\{x+\frac{u}{2}-\frac{u}{2},\left(x+\frac{u}{2}-u\right) \sqrt{\left(x+\frac{u}{2}\right)^{2}-\frac{q}{u}}\right\} d x \\
& =\int R\left\{\frac{s^{2}+\frac{q}{u}}{2 s}-\frac{u}{2},\left(\frac{s^{2}+\frac{q}{u}}{2 s}-u\right) \frac{-s^{2}+\frac{q}{u}}{2 s}\right\} \frac{s^{2}-\frac{q}{u}}{2 s^{2}} d s=\int R_{1}(s) d s .
\end{aligned}
$$

The next phase is to determine a condition for the polynomial (1.4) to have the double root $x=\frac{u}{2}$. 
Taking into account that the polynomial (1.4) has double root $x=\frac{u}{2}$, from the equation

$$
x^{4}+p x^{2}+q x+r=0
$$

a new equation in the variable $u$ is obtained,

$$
u^{4}+4 p u^{2}+8 q u+16 r=0 .
$$

Since $u$ satisfies the equations (1.5) and (2.1), it satisfies the following seven equations:

$$
\begin{aligned}
& u^{6}+4 p u^{4}+8 q u^{3}+16 r u^{2}=0, \\
& u^{6}+2 p u^{4}+2 q u^{3}=0, \\
& u^{5}+4 p u^{3}+8 q u^{2}+16 r u=0, \\
& u^{5}+2 p u^{3}+2 q u^{2}=0, \\
& u^{4}+4 p u^{2}+8 q u+16 r=0, \\
& u^{4}+2 p u^{2}+2 q u=0, \\
& u^{3}+2 p u+2 q=0 .
\end{aligned}
$$

The last system of equations, according to the Silvester's method of elimination, can be viewed as a linear homogenous system in the unknowns $u^{6}, u^{5}, u^{4}, u^{3}, u^{2}, u, 1$ which, since $u \neq 0$, should have infinite number of solutions. We easily obtain the condition for having infinite number of solutions:

$$
2 p\left|\begin{array}{cccc}
p & 3 q & 8 r & 0 \\
0 & p & 2 q & 4 r \\
0 & p & 3 q & 8 r \\
1 & 0 & 2 p & 2 q
\end{array}\right|+\left|\begin{array}{cccc}
3 q & 8 r & 0 & 0 \\
p & 3 q & 8 r & 0 \\
0 & p & 3 q & 4 r \\
1 & 0 & 2 p & q
\end{array}\right|=0
$$

which is equivalent to:

$$
4 p^{3} q^{2}-16 r p^{4}-144 r p q^{2}+128 r^{2} p^{2}+27 q^{4}-256 r^{3}=0 .
$$

Actually the relation (2.4) is the necessary condition that should be satisfied by the coefficients of the polynomial (1.4) for having double root.

Method for determining the double root of the polynomial (1.4) based on the Euclidian algorithm for greatest common devisor of two polynomials will be derived in the following lines.

When the condition (2.4) is satisfied, the polynomial (1.4) has the double root $\frac{u_{0}}{2}$ and in the same time $u_{o}$ is the double root of the polynomial $P_{4}(u)$ and the single root of the polynomial $P_{3}(u)$ :

$$
P_{4}(u)=u^{4}+4 p u^{2}+8 q u+16 r, P_{3}(u)=u^{3}+2 p u+2 q,
$$

hence

$$
u_{0}^{4}+4 p u_{0}^{2}+8 q u_{0}+16 r=0, u_{0}^{3}+2 p u_{0}+2 q=0 .
$$


Dividing the polynomials in few steps, the following is obtained:

$$
\begin{gathered}
P_{4}(u)=u P_{3}(u)+2 P_{2}(u), \text { where } P_{2}(u)=p u^{2}+3 q u+8 r \\
P_{3}(u)=\left(\frac{1}{p} u-\frac{3 q}{p^{2}}\right) P_{2}(u)+P_{1}(u), \text { where } P_{1}(u)=\left(2 p-\frac{8 r}{p}+\frac{9 q^{2}}{p^{2}}\right) u+2 q+\frac{24 q r}{p^{2}} .
\end{gathered}
$$

$P_{4}\left(u_{0}\right)=P_{3}\left(u_{0}\right)=0$ implies $P_{2}\left(u_{0}\right)=P_{1}\left(u_{0}\right)=0$, thus the double root of the polynomial (1.4), if it exists, can be obtained by the formula

$$
x=\frac{u_{0}}{2}=-\frac{p^{2} q+12 q r}{2 p^{3}-8 p r+9 q^{2}} .
$$

\section{EXAMPLES}

Example 1. The polynomial

$$
p(x)=x^{4}+x^{2}-6 x+4
$$

whose coefficients are

$$
p=1, q=-6, r=4,
$$

satisfies the condition (2.4), which means that the polynomial has a double root, that can be obtained from the formula (2.5), $x=1$. So, the presentation (1.6) has the form

$$
p(x)=x^{4}+x^{2}-6 x+4=(x-1)^{2}\left[(x+1)^{2}+3\right]=(x-1)^{2} \cdot\left(x^{2}+2 x+4\right) .
$$

Example 2. The polynomial

$$
p(x)=x^{4}-2 x^{2}-4 \sqrt{2} x+8
$$

with the coefficients

$$
p=-2, q=-4 \sqrt{2}, r=8,
$$

satisfies (2.4) and the formula (2.5) gives that the double root of the given polynomial is $x=\sqrt{2}$. The presentation (6) will take the form:

$$
p(x)=x^{4}-2 x^{2}-4 \sqrt{2} x+8=(x-\sqrt{2})^{2}\left[(x+\sqrt{2})^{2}+2\right]=(x-\sqrt{2})^{2}\left(x^{2}+2 \sqrt{2} x+4\right) .
$$

Example 3. Let us consider the differential equation

$$
\left(x^{4}+x^{3}-3 x^{2}-x+2\right) y^{\prime}-\left(4 x^{3}+3 x^{2}-6 x-1\right) y=\frac{\left(x^{4}+x^{3}-3 x^{2}-x+2\right)^{3}}{\sqrt{x^{4}-3 x+2 x}} .
$$

The polynomial $x^{4}-3 x+2 x$ is of type (1.4), with $p=-3, q=2, r=0$ and satisfies the condition (2.4). Its double root is $x=1$.

The differential equation has a general solution

$$
\begin{aligned}
y & =\left(x^{4}+x^{3}-3 x^{2}-x+2\right)\left[\int \frac{x^{4}+x^{3}-3 x^{2}-x+2}{\sqrt{x^{4}-3 x^{2}+2 x}} d x+c\right] \\
& =\left(x^{4}+x^{3}-3 x^{2}-x+2\right)\left[\int \frac{(x-1)^{2}(x+2)(x+1)}{(x-1) \sqrt{x^{2}+2 x}} d x+c\right]
\end{aligned}
$$




$$
\begin{aligned}
& =\left(x^{4}+x^{3}-3 x^{2}-x+2\right)\left[\int \frac{(x-1)(x+2)(x+1)}{\sqrt{x^{2}+2 x}} d x+c\right] \\
& =\left(x^{4}+x^{3}-3 x^{2}-x+2\right)\left[\frac{x\left(2 x^{3}+5 x^{2}-7 x-18\right)}{6 \sqrt{x^{2}+2 x}}-\arcsin \frac{\sqrt{x}}{\sqrt{2}}+c\right] .
\end{aligned}
$$

Without loss of generality we assumed that $x>1$. In solving the integral we used the technique used in calculus

$$
\int \frac{P_{n}(x)}{\sqrt{a x^{2}+b x+c}} d x=Q_{n-1}(x) \sqrt{a x^{2}+b x+c}+\lambda \int \frac{d x}{\sqrt{a x^{2}+b x+c}},
$$

where $P_{n}(x)$ is an $n$-th order polynomial, $Q_{n-1}(x)$ is an (n-1)-th order polynomial with undetermined coefficients, and $\lambda$ is undetermined parameter.

Example 4. Let us find the general solution of the differential equation:

$$
\ln (x+1) y^{\prime}-\frac{y}{x+1}=\ln ^{3}(x+1) \frac{2 x^{3}+x-3}{\sqrt{x^{4}+x^{2}-6 x+4}} .
$$

Dividing both sides of the equation by $\ln (x+1)$, we obtain the normal form of the linear differential equation of first order:

$$
y^{\prime}-\frac{y}{(x+1) \ln (x+1)}=\ln ^{2}(x+1) \frac{2 x^{3}+x-3}{\sqrt{x^{4}+x^{2}-6 x+4}} .
$$

Its general solution is

$$
y=\ln (x+1) \int \ln (x+1) \frac{2 x^{3}+x-3}{\sqrt{x^{4}+x^{2}-6 x+4}} d x .
$$

We will use integration by parts in the last integral,

$$
u=\ln (x+1), d u=\frac{d x}{x+1}, d v=\frac{2 x^{3}+x-3}{\sqrt{x^{4}+x^{2}-6 x+4}} d x=\frac{2 x^{3}+x-3}{(x-1) \sqrt{x^{2}+2 x+4}} d x, v=(x-1) \sqrt{x^{2}+2 x+4} .
$$

At this phase, the solution is

$$
y=\ln (x+1)\left[\ln (x+1)(x-1) \sqrt{x^{2}+2 x+4}-\int(x-1) \sqrt{x^{2}+2 x+4} \frac{d x}{x+1}\right] .
$$

After applying the Euler's substitution $\sqrt{x^{2}+2 x+4}=x+t$, we obtain integral of rational function and the final solution:

$$
y=\ln (x+1)\left[(\ln (x+1))(x-1) \sqrt{x^{2}+2 x+4}+\frac{13}{12}+\frac{9}{4(t-1)}-\frac{3 t}{4}-\frac{t^{2}}{4}-\frac{t^{3}}{12}+3 \ln \frac{t-1}{\left(-t^{2}+2 t+2\right)^{2}}+c\right],
$$

where $t=\sqrt{x^{2}+2 x+4}-x$.

Example 5. Let's find the general solution of the differential equation

$$
\operatorname{arctg} x y^{\prime}-\frac{y}{1+x^{2}}=\operatorname{arctg}^{2} x \sqrt{x^{4}-2 x^{2}-4 \sqrt{2} x+8} .
$$

Although the equation is not of the type (1.1) (the function $\operatorname{arctg} x$ is powered on 2 not on 3 , on the right hand side), the described method yields to the true solution. The 4-th order polynomial, as it can be seen in example 2, has a double root $x=\sqrt{2}$.

The general solution of the equation is 


$$
\begin{aligned}
y & =\operatorname{arctg} x\left[\int \sqrt{x^{4}-2 x^{2}-4 \sqrt{2} x+8} d x+c\right] \\
& =\operatorname{arctg} x\left[\int(x-\sqrt{2}) \sqrt{x^{2}+2 \sqrt{2} x+4} d x+c\right] \\
& =\operatorname{arctg} x\left[\int(x-\sqrt{2}) \sqrt{(x+\sqrt{2})^{2}+2} d x+c\right] \\
& =2 \sqrt{2} \operatorname{arctg} x\left[\sqrt{\left(\frac{x^{2}+2 \sqrt{2} x}{2}\right)^{3}}-\frac{x+\sqrt{2}}{4 \sqrt{2}} \sqrt{\frac{x^{2}+2 \sqrt{2} x}{2}}+\operatorname{arcsinh} \frac{x+\sqrt{2}}{\sqrt{2}}+c\right] .
\end{aligned}
$$

We used the substitution $x+\sqrt{2}=\sqrt{2}$ sh $t$ in solving the integral. We have here also assumed, without loss of generality, that $x>\sqrt{2}$.

\section{CONCLUSION}

A class of linear differential equation of first order, whose general solution contains pseudoeliptic integral is considered in this article. The main result gives a way how to come to the explicit form of the general solution.

\section{References}

[1] E. L. Ince, Ordinary Differential Equations, Dover Publications INC. New York 1956.

[2] Л. С. Понтрягин, Обыкновенные дифференциальные уравнения, Наука, Москва, 1970.

[3] Н. Обрешков, ВИСША АЛГЕБРА, Наука и изкуство, София, 1966.

${ }^{1}$ Faculty of Mechanical Engineering, Univ. Ss Cyril and Methodius, Skopje, Macedonia,

E-mail: lazo.dimov@mf.edu.mk

${ }^{2}$ Faculty of Electrical Engineering and Information Technologies, Univ. Ss Cyril and

Methodius, Skopje, Macedonia,

E-mail: borom@feit.ukim.edu.mk

${ }^{3}$ University for Information Science and Technology "St. Paul the Apostole", Ohrid,

Macedonia,

E-mail: elena.hadzieva@uist.edu.mk 